\title{
CREENCIAS IRRACIONALES EN ESTUDIANTES DEL NIVEL SECUNDARIO DE UN CENTRO DE EDUCACIÓN BÁSICA ALTERNATIVA
}

\author{
Irrational beliefs in secondary school students from a center for \\ alternative basic education
}

\author{
Omar Gibrahim Ortiz Álvarez* \\ Universidad Nacional Mayor de San Marcos - Lima, Perú \\ https://orcid.org/0000-0002-7126-3428
}

\begin{abstract}
Resumen
El objetivo del presente artículo es determinar las diferencias en las creencias irracionales en estudiantes del nivel secundario de un Centro de Educación Básica Alternativa, según género y grupo etario. El estudio es de nivel descriptivo y diseño descriptivo comparativo. La muestra fue obtenida por técnica censal, quedando conformada por 161 estudiantes de ambos géneros del primer al cuarto grado del nivel secundario. Se administró el Inventario de Creencias irracionales de Albert Ellis. Se calcularon frecuencias y porcentajes, y se emplearon la prueba de normalidad de Kolmogorov-Smirnov y las pruebas U de MannWithney y Kruskal-Wallis para la constrastación de las hipótesis. El análisis mostró que las diferencias en creencias irracionales, teniendo en cuenta el género, no son significativas al nivel de $p<0,05$, a excepción de la creencia irracional: Dependencia $(U=1,687,000$; Sig. unilateral $=0,036)$, con rango promedio mayor en el grupo de varones. Asimismo, los resultados evidencian que, de acuerdo al grupo etario, no se registran diferencias significativas, al nivel de $p<0,05$, en las creencias irracionales, excepto Evitación de problemas $(X 2=7,017 ;$ Sig. $=0,030)$, con rango promedio mayor en el grupo de estudiantes con edades de 20 a 29. En conclusión, se establece que, respecto al género, aunque en la mayor parte de las creencias irracionales hay una ligera tendencia a ser mayor en las estudiantes mujeres, solo se aprecia diferencia significativa en la creencia irracional: Dependencia; mientras que, por el grupo etario, la diferencia significativa solo se registra en la creencia irracional: Evitación de problemas.
\end{abstract}

Palabras clave: Creencias irracionales, estudiantes, secundaria, Centro de Educación Básica Alternativa.

\begin{abstract}
The objective of this article is to determine the differences in irrational beliefs among secondary school students from a Center for Alternative Basic Education, according to gender and age group. This is a descriptive study with a comparative descriptive design. The population was obtained through a census, and is made up of 161 students of both sexes from the first to the fourth grade of secondary school. The Albert Ellis Irrational Belief Inventory was administered. Frequencies and percentages were calculated, and the Kolmogorov-Smirnov normality test and the Mann-Whitney and Kruskal-Wallis U tests were used to test the hypotheses. The analysis showed that the differences in irrational beliefs, considering gender, are not significant at the level of $p<0.05$, except for one irrational belief: dependence $(U=1.687 .000$; Unilateral sig. $=0.036)$, with a higher average rank in the male group. Likewise, the results show that, according to the age group, there are no significant differences, at the level of $p<0.05$, in irrational beliefs, except problem avoidance $(X 2=7.017 ;$ Sig. $=0.030)$, with a higher average rank in the group of students aged 20 to 29. In conclusion, with respect to gender, although most irrational beliefs have a slight tendency to be higher in female students, the only significant difference is noted in irrational belief: dependence. In contrast, with respect to age group, significant differences are only found for the irrational belief: problem avoidance.
\end{abstract}

Keywords: irrational beliefs, students, high school, Alternative Basic Education Center.

*Psicólogo.psico_mind@hotmail.com 


\section{INTRODUCCIÓN}

Al nivel secundario de un Centro de Educación Básica Alternativa (CEBA) concurren tanto estudiantes adolescentes, como estudiantes jóvenes y adultos, constituyendo los adolescentes cerca del 50\% del alumnado. Respecto a la institución educativa, Otero (2001) señala que es el lugar más importante de socialización durante la etapa de la adolescencia. Se trata de un contexto de interrelación donde aprenden importantes pautas de acercamiento y aceptación con sus pares y con figuras de autoridad, como son los profesores. Las condiciones en que se sienten los adolescentes con respecto a sí mismos, influyen de manera significativa en las experiencias académicas, familiares, laborales y, fundamentalmente, en las relaciones interpersonales, así como también las respuestas y la toma de decisiones que pueden tener frente a un acontecimiento. Precisamente, estas interrrelaciones y las exigencias académicas y laborales, pueden alentar la generación de ideas irracionales o, en todo caso, su consolidación como tales, que, como afirma Ellis (1998), estas creencias se forman a lo largo de la vida. Si estas creencias son racionales, ayudan al estudiante a sobrellevar las adversidades académicas, consiguiendo muchas veces sus objetivos; y, por el contrario, si estas creencias son de naturaleza ilógica e irracionales, traen consigo desajustes emocionales que, a su vez, se manifiestan en comportamientos inadecuados que podrían perjudicar e impactar en la educación del alumno, así como también en el desenvolvimiento del docen

Ellis y Grieger (1981) afirman que las creencias irracionales son afirmaciones no empíricas o irreales que uno mismo se plantea, donde influyen aspectos innatos como aprendidos de cada persona. Son "suprageneralizaciones" obtenidas de los datos que se observan. Asimismo, señalan que las creencias irracionales no solo se derivan de la predisposición personal del individuo a percibir el entorno con ciertas distorsiones y a formular aseveraciones poco veraces sobre lo sucedido o lo que le sucederá con probabilidad; también provienen de sus razonamientos categóricos acerca de que "le debería suceder" o "le tiene que pasar" para que, de esta forma, le sea posible lograr perentoriamente lo que desea; pero una vez que sus deseos se vuelven en requerimientos obsesivos, tales razonamientos poco realistas surgen casi inexorablemente y, a menudo, en gran medida.

Ellis (1998) agrupó en torno a 10 todas las ideas irracionales básicas de la civilización occidental actual, como son: Necesidad de aprobación, referida a aquella de sentirse amado y aprobado por toda persona de su entorno; altas autoexpectativas, en la que se considera a sí mismo valioso y, por lo tanto, muy competente, suficiente y capaz de lograr cualquier cosa en todos los aspectos posibles; culpabilización, en la que cierta clase de personas viles, malvadas e infames deben ser seriamente culpabilizadas y castigadas por su maldad; intolerancia a la frustración, cuando las cosas no van por el camino que a uno le gustaría que fuesen; miedo $o$ ansiedad, donde uno debe sentirse muy inquieto por algo que es o puede ser peligroso o terrible, debiendo pensar constantemente en la posibilidad de que ocurra; causas externas, en la que la desgracia humana se origina por tales causas y la gente tiene muy poca capacidad o ninguna para controlar sus penas y perturbaciones; evitación de problemas, que indica que es más fácil evitar que afrontar ciertas responsabilidades y dificultades en la vida; dependencia, en la medida en que se debe depender de los demás y se necesita a alguien más fuerte en quien confiar; indefensión, en la que la historia pasada de uno es un determinante y decisiva de la conducta actual, pues algo que ocurrió una vez y le conmocionó debe seguir afectándole indefinidamente; y pasividad, pues hay que lograr la felicidad por inercia y sin hacer nada, o "divertirse" solo y pasivamente.

El ser humano, desde que se va desarrollando biológicamente, también va generando creencias que pueden ser racionales, irracionales o ambas, que, dependiendo del contexto en el que se desarrolle, tenderán a aumentar, mantener o disminuir; en tal sentido, se puede decir que son las creencias irracionales aquellas que pueden llegar a obstaculizar o limitar el bienestar y tranquilidad del individuo (Carbonero et al., 2010). En tal sentido, Cartagena (2011) indica que las creencias irracionales son conocimientos evaluativos de carácter personal y tiene dos características: por un lado, son absolutas o innegables por su naturaleza y por otro provocan emociones negativas que interfieren en la persecución y obtención de metas y consecuentemente de conductas disfuncionales como la depresión, ansiedad, culpabilidad, miedo, aislamiento, demora, 
entre otras. Además, en otro estudio refirió que los adolescentes con pensamientos irracionales pueden tener alteraciones en su autoestima, destruyendo sus capacidades y empobreciendo las emociones, que se acompañan de una escasa, pobre o nula valoración de sí mismos, lo cual producirá en el adolescente un bajo rendimiento académico, poco desarrollo de sus habilidades sociales, refugio en el alcohol y drogas, entre otras.

Se consideró necesario elegir la temática de las creencias irracionales en un CEBA, por el desenvolvimiento que tiene en la educación básica, donde se ha podido apreciar la forma en que se presenta, y es por ello, que surge la necesidad de profundizar más desde diversas perspectivas para conseguir una mejor comprensión y afrontamiento. Las creencias irracionales, ya nombradas, se manifiestan de manera presente en los estudiantes de los CEBA que, al manifestarse en diversos comportamientos antisociales, dan una mayor riqueza para poderlo estudiar. En ese este contexto, en el Perú, los CEBA no se encuentran ajenos a la problemática relacionada con conductas antisociales, disfunción familiar, conflictos de pareja, consumo de alcohol y drogas, teniendo en cuenta que su población de estudiantes viene con una historia inconclusa de vida escolar. La cual se caracteriza por ser estar conformada por adolescentes, jóvenes y adultos, de ambos géneros, cuyas edades fluctúan entre los 14 a 39 años; caracterizándose estos CEBA, además, por albergar a estudiantes que habitualmente han repetido el año escolar o no han podido estudiar en su momento, en los grados regulares.

También se estimó pertinente establecer las diferenciaciones de acuerdo al género y el grupo etario; es decir, si por la cultura de género en la cual se han criado y desarrollado los estudiantes del CEBA que forma parte del estudio, pueden existir diferencias en las creencias irracionales entre varones y mujeres, y si además el ajuste de personalidad y la consolidación de la identidad, conforme transcurre el tiempo, puede reducir o favorecer la presencia de creencias irracionales entre estudiantes adolescentes, jóvenes y adultos. De ese modo, los resultados permitirían precisar las medidas correctivas a utilizarse como parte de una intervención educativa temprana.
El objetivo general es el determinar si existen diferencias en las creencias irracionales en estudiantes de un Centro de Educación Básica Alternativa, según el género y el grupo etario.

\section{MÉTODO}

\section{Nivel y diseño}

Este estudio corresponde al nivel de investigación descriptiva y al diseño descriptivo comparativo (Sánchez y Reyes, 2015).

\section{Participantes}

La población estuvo integrada por 161 estudiantes (128 varones y 33 mujeres) matriculados entre los grados primero y cuarto del nivel secundario de un Centro de Educación Básica Alternativa de Lima Metropolitana.

La muestra se seleccionó mediante la técnica censal, que consiste en incluir al 100\% de los integrantes de la población, de modo que todas las unidades de análisis de la población son estimadas como muestra (Ramírez, 1997). Por consiguiente, quedó conformada por 161 estudiantes del nivel secundario de un CEBA de Lima Metropolitana.

El grupo de investigación se ha dividido, de acuerdo al género, en estudiantes varones y estudiantes mujeres, y en base a su edad cronológica, que varía de los 14 a 39 años.

\section{Instrumento}

Inventario de Creencias Irracionales de Albert Ellis

Elaborado por Albert Ellis en 1980 y traducido al castellano por McKay y Navas (2008), el Inventario se aplica tanto a adolescentes como a adultos, de modo individual o colectivo. Su tiempo de aplicación es de 20 a 30 minutos. Permite identificar las creencias irracionales que han sido asumidas por el individuo. Los ítems que lo conforman están enumerados del 1 al 100, agrupados en bloques de diez grupos, en la que cada uno de ellos comprende diez preguntas con opciones de respuesta dicotómicas (Sí-No). Cada uno de los diez bloques corresponde a una dimensión que agrupa los reactivos sobre una determinada creencia 
irracional. Así, se han categorizado de la siguiente manera: Necesidad de aprobación (creencia I); altas autoexpectativas (creencia II); culpabilización (creencia III); intolerancia a la frustración (creencia IV); causas externas (creencia V); miedo o ansiedad (creencia VI); evitación de problemas (creencia VII); dependencia (creencia VIII); indefensión (creencia IX); y pasividad (creencia X).

Para la calificación, se realiza la sumatoria de cada creencia individual, la cual se obtiene con el puntaje total para cada una de ellas. De esa manera, el puntaje obtenido en un rango de 0 a 5 es considerado un puntaje promedio o bajo; en un rango de 6 a 7 se le estima como moderado, en el que, si bien el sujeto posee la creencia irracional, esta puede ser ocasionante del algún problema conductual o emocional; y si el puntaje varía en el rango de 8 a 10, entonces corresponde a un elevado nivel de la creencia irracional, lo que estaría ocasionando un trastorno emocional.

Para establecer la validez del Inventario, Tang (2011) empleó el criterio de jueces expertos, quienes asignaron sus valores, y a partir de éstos se calculó la $\mathrm{V}$ de Aiken, obteniéndose los siguientes resultados: Creencia I, V = 0,93; creencia II, V = 1,00; creencia III, $\mathrm{V}=0,80$; creencia $\mathrm{IV}, \mathrm{V}=0,90$; creencia $\mathrm{V}, \mathrm{V}=0,93$; creencia VI, V =0,98; creencia VII, V =0,98; creencia VIII, V = 0,97; creencia IX, V = 0,94; y creencia X, V $=0,98$. Tales coeficientes $\mathrm{V}$ de Aiken indican que el instrumento presenta un alto nivel de validez y, por lo tanto, es idóneo para su administración a la muestra de estudiantes seleccionada.

Respecto a la confiabilidad, Kuba (2017) realizó una actualización partiendo de un análisis de la técnica de consistencia interna mediante el coeficiente alfa de Cronbach, consiguiendo un valor equivalente a 0.81 , el cual indica que los ítems del instrumento poseen un alto nivel de consistencia interna y, en consecuencia, el Inventario de Creencias Irracionales es confiable y apto para su aplicación a la muestra de estudio.

\section{RESULTADOS}

Creencias irracionales según el género

Se observa en la tabla 1, que entre los estudiantes varones y mujeres, por lo general, los porcentajes son bastantes similares, salvo algunas excepciones, las que a continuación se detallan: En la creencia irracional: Intolerancia a la frustración, se aprecia en el nivel promedio o bajo una mayor proporción de varones $(39,8 \%)$ que de mujeres $(30,3 \%)$; en cambio, en la creencia irracional: Dependencia, es mayor el porcentaje de mujeres $(48,5 \%)$ que el de varones $(32,0 \%)$, también en el nivel promedio o bajo; pasando al nivel moderado o medio, se registran dos marcadas diferencias, como en la creencia irracional: Necesidad de aprobación, donde el porcentaje de varones $(19,5 \%)$ es notoriamente mayor que el de mujeres $(9,1 \%)$, y en la creencia irracional: Intolerancia a la frustración, cuyo porcentaje de mujeres $(36,4 \%)$ es bastante mayor al de varones $(21,9 \%)$; y siguiendo con el nivel alto, se advierte en la creencia irracional: Dependencia que los varones $(37,5 \%)$ representan un mayor porcentaje que el de las mujeres $(27,3 \%)$, y, de modo similar, en la creencia irracional: Evitación de problemas, el porcentaje de varones $(35,9 \%)$ es claramente más visible que el de mujeres $(27,3 \%)$. 
Tabla 1

Frecuencias y porcentajes en las creencias irracionales según el género

\begin{tabular}{|c|c|c|c|c|c|c|c|c|c|}
\hline \multirow{2}{*}{ Creencia irracional } & \multirow{2}{*}{ Género } & \multicolumn{2}{|c|}{ Promedio o bajo } & \multicolumn{2}{|c|}{ Moderado o medio } & \multicolumn{2}{|c|}{ Alto } & \multicolumn{2}{|c|}{ Total } \\
\hline & & $\mathrm{F}$ & $\%$ & $\mathrm{~F}$ & $\%$ & $\mathrm{~F}$ & $\%$ & $\mathrm{~F}$ & $\%$ \\
\hline \multirow[t]{2}{*}{ Necesidad de aprobación } & M & 69 & 53,9 & 25 & 19,5 & 34 & 26,6 & 128 & 100,0 \\
\hline & F & 19 & 57,6 & 3 & 9,1 & 11 & 33,3 & 33 & 100,0 \\
\hline \multirow[t]{2}{*}{ Altas auto- expectativas } & M & 51 & 39,8 & 29 & 22,7 & 48 & 37,5 & 128 & 100,0 \\
\hline & F & 12 & 36,4 & 10 & 30,3 & 11 & 33,3 & 33 & 100,0 \\
\hline \multirow[t]{2}{*}{ Culpabilización } & M & 53 & 41,4 & 30 & 23,4 & 45 & 35,2 & 128 & 100,0 \\
\hline & $\mathrm{F}$ & 13 & 39,4 & 10 & 30,3 & 10 & 30,3 & 33 & 100,0 \\
\hline \multirow[t]{2}{*}{ Intolerancia a la frustración } & M & 51 & 39,8 & 28 & 21,9 & 49 & 38,3 & 128 & 100,0 \\
\hline & $\mathrm{F}$ & 10 & 30,3 & 12 & 36,4 & 11 & 33,3 & 33 & 100,0 \\
\hline \multirow[t]{2}{*}{ Causas externas } & M & 54 & 42,2 & 30 & 23,4 & 44 & 34,4 & 128 & 100,0 \\
\hline & F & 14 & 42,4 & 8 & 24,2 & 11 & 33,3 & 33 & 100,0 \\
\hline \multirow[t]{2}{*}{ Miedo o ansiedad } & M & 51 & 39,8 & 27 & 21,1 & 50 & 39,1 & 128 & 100,0 \\
\hline & F & 11 & 33,3 & 9 & 27,3 & 13 & 39,4 & 33 & 100,0 \\
\hline \multirow[t]{2}{*}{ Evitación de problemas } & M & 51 & 39,8 & 31 & 24,2 & 46 & 35,9 & 128 & 100,0 \\
\hline & $\mathrm{F}$ & 14 & 42,4 & 10 & 30,3 & 9 & 27,3 & 33 & 100,0 \\
\hline \multirow[t]{2}{*}{ Dependencia } & M & 41 & 32,0 & 39 & 30,5 & 48 & 37,5 & 128 & 100,0 \\
\hline & F & 16 & 48,5 & 8 & 24,2 & 9 & 27,3 & 33 & 100,0 \\
\hline \multirow[t]{2}{*}{ Indefensión } & M & 48 & 37,5 & 35 & 27,3 & 45 & 35,2 & 128 & 100,0 \\
\hline & F & 10 & 30,3 & 9 & 27,3 & 14 & 42,4 & 33 & 100,0 \\
\hline \multirow[t]{2}{*}{ Pasividad } & M & 44 & 34,4 & 37 & 28,9 & 47 & 36,7 & 128 & 100,0 \\
\hline & F & 9 & 27,3 & 11 & 33,3 & 13 & 39,4 & 33 & 100,0 \\
\hline
\end{tabular}

\section{Creencias irracionales según el grupo etario}

Se aprecia en la tabla 2, en el nivel promedio o bajo, una tendencia a que, en varias creencias irracionales, se presente un mayor porcentaje en el grupo etario de 30 a 39 años; observándose una marcada diferencia con los otros grupos etarios en las creencias irracionales: Causas externas $(62,5 \%)$ y Dependencia (también 62,5\%), evidenciándose, en cambio, en la creencia irracional: Indefensión, un mayor porcentaje en el grupo etario de 14 a 19 años $(43,0 \%)$. Mientras que en el nivel moderado o medio se registra, en la creencia irracional: Culpabilización, el 50,0\% en el grupo etario de 30 a 39 años, mayor en comparación con los otros grupos etarios; lo mismo se observa en las creencias irracionales: Evitación de problemas e Indefensión, con 50,0\% por igual en ambos, en el grupo etario de 30 a 39 años; por el contrario, siempre en el nivel moderado o medio, se advierte un mayor porcentaje en el grupo etario de 14 a 19 años, correspondiente a las creencias irracionales: Intolerancia a la frustración $(29,1 \%)$, Causas externas $(22,8 \%)$ y Pasividad $(31,6 \%)$. 


\section{Tabla 2}

Frecuencias y porcentajes en las creencias irracionales según el grupo etario

\begin{tabular}{|c|c|c|c|c|c|c|c|c|c|}
\hline \multirow[t]{2}{*}{ Creencia irracional } & \multirow{2}{*}{ Grupo etario } & \multicolumn{2}{|c|}{ Promedio o bajo } & \multicolumn{2}{|c|}{ Moderado o medio } & \multicolumn{2}{|c|}{ Alto } & \multicolumn{2}{|c|}{ Total } \\
\hline & & $\mathrm{F}$ & $\%$ & $\mathrm{~F}$ & $\%$ & $\mathrm{~F}$ & $\%$ & $\mathrm{~F}$ & $\%$ \\
\hline \multirow[t]{3}{*}{ Necesidad de aprobación } & $14-19$ a & 47 & 59,5 & 12 & 15,2 & 20 & 25,3 & 79 & 100,0 \\
\hline & $20-29$ a & 36 & 48,6 & 15 & 20,3 & 23 & 31,1 & 74 & 100,0 \\
\hline & $30-39$ a & 5 & 62,5 & 1 & 12,5 & 2 & 25,0 & 8 & 100,0 \\
\hline \multirow[t]{3}{*}{ Altas auto- expectativas } & $14-19$ a & 30 & 38,0 & 21 & 26,6 & 28 & 35,4 & 79 & 100,0 \\
\hline & $20-29$ a & 31 & 41,9 & 15 & 20,3 & 28 & 37,8 & 74 & 100,0 \\
\hline & $30-39$ a & 2 & 25,0 & 3 & 37,5 & 3 & 37,5 & 8 & 100,0 \\
\hline \multirow[t]{3}{*}{ Culpabilización } & $14-19 \mathrm{a}$ & 29 & 36,7 & 22 & 27,8 & 28 & 35,4 & 79 & 100,0 \\
\hline & $20-29$ a & 33 & 44,6 & 14 & 18,9 & 27 & 36,5 & 74 & 100,0 \\
\hline & $30-39$ a & 4 & 50,0 & 4 & 50,0 & 0 & 0,0 & 8 & 100,0 \\
\hline \multirow[t]{3}{*}{ Intolerancia a la frustración } & $14-19$ a & 28 & 35,4 & 23 & 29,1 & 28 & 35,4 & 79 & 100,0 \\
\hline & $20-29$ a & 29 & 39,2 & 16 & 21,6 & 29 & 39,2 & 74 & 100,0 \\
\hline & $30-39 a$ & 4 & 50,0 & 1 & 12,5 & 3 & 37,5 & 8 & 100,0 \\
\hline \multirow[t]{3}{*}{ Causas externas } & $14-19$ a & 33 & 41,8 & 18 & 22,8 & 28 & 35,4 & 79 & 100,0 \\
\hline & $20-29$ a & 30 & 40,5 & 19 & 25,7 & 25 & 33,8 & 74 & 100,0 \\
\hline & $30-39$ a & 5 & 62,5 & 1 & 12,5 & 2 & 25,0 & 8 & 100,0 \\
\hline \multirow[t]{3}{*}{ Miedo o ansiedad } & $14-19$ a & 30 & 38,0 & 16 & 20,3 & 33 & 41,8 & 79 & 100,0 \\
\hline & $20-29$ a & 28 & 37,8 & 19 & 25,7 & 27 & 36,5 & 74 & 100,0 \\
\hline & $30-39 a$ & 4 & 50,0 & 1 & 12,5 & 3 & 37,5 & 8 & 100,0 \\
\hline \multirow[t]{3}{*}{ Evitación de problemas } & $14-19 \mathrm{a}$ & 38 & 48,1 & 20 & 25,3 & 21 & 26,6 & 79 & 100,0 \\
\hline & $20-29$ a & 24 & 32,4 & 17 & 23,0 & 33 & 44,6 & 74 & 100,0 \\
\hline & $30-39$ a & 3 & 37,5 & 4 & 50,0 & 1 & 12,5 & 8 & 100,0 \\
\hline \multirow[t]{3}{*}{ Dependencia } & 14-19 a & 25 & 31,6 & 24 & 30,4 & 30 & 38,0 & 79 & 100,0 \\
\hline & $20-29 a$ & 27 & 36,5 & 21 & 28,4 & 26 & 35,1 & 74 & 100,0 \\
\hline & $30-39$ a & 5 & 62,5 & 2 & 25,0 & 1 & 12,5 & 8 & 100,0 \\
\hline \multirow[t]{3}{*}{ Indefensión } & $14-19$ a & 34 & 43,0 & 20 & 25,3 & 25 & 31,6 & 79 & 100,0 \\
\hline & $20-29$ a & 22 & 29,7 & 20 & 27,0 & 32 & 43,2 & 74 & 100,0 \\
\hline & $30-39$ a & 2 & 25,0 & 4 & 50,0 & 2 & 25,0 & 8 & 100,0 \\
\hline \multirow[t]{3}{*}{ Pasividad } & 14-19 a & 30 & 38,0 & 25 & 31,6 & 24 & 30,4 & 79 & 100,0 \\
\hline & $20-29$ a & 20 & 27,0 & 22 & 29,7 & 32 & 43,2 & 74 & 100,0 \\
\hline & $30-39$ a & 3 & 37,5 & 1 & 12,5 & 4 & 50,0 & 8 & 100,0 \\
\hline
\end{tabular}




\section{Diferencias en las creencias irracionales de acuerdo al género}

Como se puede ver en la tabla 3, las diferencias entre los rangos promedio en las creencias irracionales entre varones y mujeres arrojan valores de $\mathrm{U}$ de Mann-Withney estadísticamente no significativos al nivel de $\mathrm{p}<0,05$, a excepción de la creencia irracional: Dependencia, donde el valor U de Mann-Withney obtenido ( $\mathrm{U}=1,687,000)$ es significativo al nivel de $\mathrm{p}<0,05$, registrándose un mayor rango promedio en el grupo de estudiantes varones $(84,32)$.

\section{Tabla 3}

Resultados con la U de Mann-Withney de la comparación, según género, de los rangos promedio en las creencias irracionales

\begin{tabular}{|c|c|c|c|c|c|}
\hline Creencia irracional & Género & $\mathrm{N}$ & Rango promedio & $\mathrm{U}$ & Sig. unilateral \\
\hline Necesidad de aprobación & $\begin{array}{c}\mathrm{M} \\
\mathrm{F}\end{array}$ & $\begin{array}{r}128 \\
33\end{array}$ & $\begin{array}{l}80,63 \\
82,42\end{array}$ & $2,065,000$ & 0,419 \\
\hline Altas auto- expectativas & $\begin{array}{c}\mathrm{M} \\
\mathrm{F}\end{array}$ & $\begin{array}{r}128 \\
33\end{array}$ & $\begin{array}{l}81,07 \\
80,74\end{array}$ & $2,103,500$ & 0,486 \\
\hline Culpabilización & $\begin{array}{r}\mathrm{M} \\
\mathrm{F}\end{array}$ & $\begin{array}{r}128 \\
33\end{array}$ & $\begin{array}{l}80,92 \\
81,32\end{array}$ & $2,101,500$ & 0,482 \\
\hline Intolerancia a la frustración & $\begin{array}{r}\mathrm{M} \\
\mathrm{F}\end{array}$ & $\begin{array}{r}128 \\
33\end{array}$ & $\begin{array}{l}80,14 \\
84,33\end{array}$ & $2,002,000$ & 0,321 \\
\hline Causas externas & $\begin{array}{c}\mathrm{M} \\
\mathrm{F}\end{array}$ & $\begin{array}{r}128 \\
33\end{array}$ & $\begin{array}{l}80,84 \\
81,64\end{array}$ & $2,091,000$ & 0,465 \\
\hline Miedo o ansiedad & $\begin{array}{c}\mathrm{M} \\
\mathrm{F}\end{array}$ & $\begin{array}{r}128 \\
33\end{array}$ & $\begin{array}{l}79,67 \\
86,17\end{array}$ & $1,941,500$ & 0,236 \\
\hline Evitación de problemas & $\begin{array}{r}\mathrm{M} \\
\mathrm{F}\end{array}$ & $\begin{array}{r}128 \\
33\end{array}$ & $\begin{array}{l}81,66 \\
78,44\end{array}$ & $2,027,500$ & 0,360 \\
\hline Dependencia & $\begin{array}{r}\mathrm{M} \\
\mathrm{F}\end{array}$ & $\begin{array}{r}128 \\
33\end{array}$ & $\begin{array}{l}84,32 \\
68,12\end{array}$ & $1,687,000^{*}$ & 0,036 \\
\hline Indefensión & $\begin{array}{r}\mathrm{M} \\
\mathrm{F}\end{array}$ & $\begin{array}{r}128 \\
33\end{array}$ & $\begin{array}{l}79,61 \\
86,39\end{array}$ & $1,934,000$ & 0,226 \\
\hline Pasividad & $\begin{array}{c}\mathrm{M} \\
\mathrm{F}\end{array}$ & $\begin{array}{r}128 \\
33\end{array}$ & $\begin{array}{l}79,72 \\
85,95\end{array}$ & $1,948,500$ & 0,244 \\
\hline
\end{tabular}

* Significativo al nivel de $\mathrm{p}<0,05$.

Diferencias en las creencias irracionales de acuerdo al grupo etario

La tabla 4 muestra que los valores U de MannWithney obtenidos entre los rangos promedio en las creencias irracionales, según el grupo etario, no son estadísticamente significativos al nivel de $\mathrm{p}<0,05$, excepto la creencia irracional: Evitación de problemas $(\mathrm{U}=7,017)$, que presenta diferencia significativa y mayor rango promedio en los estudiantes de 20 a 29 años. 


\section{Tabla 4}

Resultados con la U de Mann-Withney de la comparación, según grupo etario, de los rangos promedio en las creencias irracionales

\begin{tabular}{|c|c|c|c|c|c|}
\hline Creencia irracional & Grupo etario & $\mathrm{N}$ & Rango promedio & Chi cuadrado & Sig. \\
\hline \multirow[t]{3}{*}{ Necesidad de aprobación } & $14-19$ a & 79 & 80,56 & & \\
\hline & $20-29$ a & 74 & 84,10 & 2,736 & 0,255 \\
\hline & $30-39$ a & 8 & 56,69 & & \\
\hline \multirow[t]{4}{*}{ Altas autoexpectativas } & $14-19$ a & 79 & 80,34 & & \\
\hline & $20-29$ a & 74 & 81,46 & 0,044 & 0,978 \\
\hline & $30-39$ a & 8 & 83,31 & & \\
\hline & $14-19$ a & 79 & 81,37 & & \\
\hline \multirow[t]{2}{*}{ Culpabilización } & $20-29$ a & 74 & 82,66 & 1,474 & 0,479 \\
\hline & $30-39$ a & 8 & 61,94 & & \\
\hline \multirow[t]{4}{*}{ Intolerancia a la frustración } & $14-19$ a & 79 & 79,56 & & \\
\hline & $20-29$ a & 74 & 82,63 & 0,171 & 0,918 \\
\hline & $30-39$ a & 8 & 80,13 & & \\
\hline & $14-19$ a & 79 & 83,00 & & \\
\hline \multirow[t]{2}{*}{ Causas externas } & $20-29$ a & 74 & 80,65 & 1,182 & 0,554 \\
\hline & $30-39$ a & 8 & 64,50 & & \\
\hline \multirow[t]{3}{*}{ Miedo o ansiedad } & $14-19$ a & 79 & 83,58 & & \\
\hline & $20-29 a$ & 74 & 79,64 & 0,924 & 0,630 \\
\hline & $30-39$ a & 8 & 68,19 & & \\
\hline \multirow[t]{4}{*}{ Evitación de problemas } & 14-19 a & 79 & 72,11 & & \\
\hline & $20-29$ a & 74 & 91,41 & $7,017 *$ & 0,030 \\
\hline & $30-39$ a & 8 & 72,44 & & \\
\hline & $14-19$ a & 79 & 84,01 & & \\
\hline \multirow[t]{3}{*}{ Dependencia } & $20-29$ a & 74 & 80,90 & 3,447 & 0,178 \\
\hline & $30-39$ a & 8 & 52,25 & & \\
\hline & $14-19$ a & 79 & 74,17 & & \\
\hline \multirow[t]{3}{*}{ Indefensión } & $20-29$ a & 74 & 87,84 & 3,427 & 0,180 \\
\hline & $30-39$ a & 8 & 85,19 & & \\
\hline & $14-19$ a & 79 & 76,03 & & \\
\hline \multirow[t]{2}{*}{ Pasividad } & $20-29$ a & 74 & 85,54 & 1,830 & 0,401 \\
\hline & $30-39$ a & 8 & 88,06 & & \\
\hline
\end{tabular}

** Significativo al nivel de $\mathrm{p}<0,01$.

\section{DISCUSIÓN}

Los resultados obtenidos fueron analizados en función a los objetivos propuestos, los cuales están referidos a las diferencias en las creencias irracionales en estudiantes de un Centro de Educación Básica Alternativa (CEBA). En términos generales, en las creencias irracionales se puede observar un nivel promedio o bajo donde se agrupa la mayor parte 
de los estudiantes; a diferencia de los resultados encontrados por Castillo (2017), quien señala que las creencias irracionales generales se sitúan en un nivel alto. En lo que respecta a las creencias irracionales de necesidad de aprobación, altas autoexpectativas, culpabilización, intolerancia a la frustración, causas externas, miedo o ansiedad, evitación de problemas, y dependencia, se encontró que se ubican en un nivel promedio predominante; a diferencia de Rivas (2016), que observó la creencia irracional: causas externas en un nivel alto, y de Olaya et al. (2014), que señalan que la necesidad de aprobación se halla en un nivel alto, así como la creencia que corresponde a la denominada como perfeccionismo.

Por otra parte, lo hallado en la creencia irracional: culpabilización se corrobora con los resultados obtenidos por López (2015) y por Rivas (2016), quienes verifican que dicha creencia tiene un nivel alto. Por lo general, en la mayoría de las creencias irracionales, es en las mujeres donde el rango promedio es ligeramente mayor al de los varones, lo cual indica que las mujeres tienen una mayor tendencia a presentar creencias irracionales que los varones, pero con una diferencia no significativa; hallazgo que discrepa con el estudio del investigador Caso (2018), que señala que es el género masculino el que tiene un mayor nivel en creencias irracionales.

En relación a la creencia irracional: necesidad de aprobación, los varones tienen un rango promedio de 80,63 , y las mujeres, un rango promedio de 82,42; lo cual evidencia que las mujeres tienen una mayor necesidad de aprobación, pero sin una diferenciación que sea significativa. En este sentido, de acuerdo a Pervin (1996), el resultado obtenido en la investigación puede deberse a que las mujeres tienen la creencia de que deben ser aceptadas y aprobadas por cuantas personas conozcan y que, al no concretarse esta creencia, se sienten ensimismadas y culpables.

Con referencia a la creencia irracional: altas autoexpectativas, los varones tienen un rango de 81,07 , en tanto que las mujeres se ubican con un rango de 80,74; estos resultados muestran que los varones tienen una puntuación levemente mayor en altas autoexpectativas. De acuerdo a Ellis (1998), este resultado podría deberse a que los varones se encuentran en un ambiente educativo competitivo, lo que podría provocar un elevado nivel de estrés, así como sentimientos de inferioridad.

En relación a la creencia irracional: culpabilización, en el género masculino se presenta un rango promedio de 80,92 , mientras que en el género femenino se presenta un rango promedio de 81,32, ligeramente mayor al de los estudiantes varones. Relativo a la creencia irracional: intolerancia a la frustración, en el género masculino se evidencia un rango promedio de 80,14 , en tanto que en el género femenino el rango promedio es de 84,33 , levemente mayor al de los varones. Concerniente a la creencia irracional: causas externas, en el género masculino se presenta un rango promedio de 80,84 , mientras que en el género femenino se registra un rango promedio de 81,64 , lo cual se traduce en una mayor tendencia en las estudiantes mujeres. En cuanto a la creencia irracional: miedo o ansiedad, en varones se verifica un rango promedio de 79,67 , y en mujeres se constata un rango promedio de 86,17 , siendo evidente una mayor tendencia en el género femenino. Con respecto a esta creencia irracional, los resultados sobre porcentajes indican que en el nivel alto se ubica el $39,1 \%$ de los varones y el 39,4\% de las mujeres, no percibiéndose diferencia enre ambos grupos, hallazgo que coincide con lo reportado por López (2015), quien señala que ambos géneros presentan un nivel alto de miedo o ansiedad ante cualquier cosa peligrosa o desconocida.

Con referencia a la creencia irracional: evitación de problemas, en el género masculino el rango promedio es de 81,66 , mientras que en el género femenino el rango promedio es de 78,44, lo cual remite a una mayor tendencia en el género masculino. En relación a la creencia irracional: dependencia, en el género masculino el rango promedio es de 84,32 , y en el género femenino el rango promedio es de 68,12 . Cabe señalar que esta es la única creencia irracional donde la diferencia sí es significativa al nivel de $\mathrm{p}<0,05$, registrándose un mayor rango promedio en los estudiantes varones, que estaría evidenciando una mayor dependencia en el género masculino. Correspondiente a la creencia irracional: indefensión, en el género masculino el rango promedio es de 79,61, y en el género femenino el rango promedio es de 86,39; se colige, entonces, que la tendencia a la indefensión es 
mayor en el género femenino, aunque la diferenciación no es significativa. Y, finalmente, acerca de la creencia irracional: pasividad, en el género masculino el rango promedio es de 79,72, y en el género femenino el rango promedio es de 85,95; registrándose, en consecuencia, una mayor tendencia a la pasividad en las estudiantes mujeres.

De otro lado, en las comparaciones de creencias irracionales de acuerdo al grupo etario, los hallazgos muestran que solo se registró diferencia significativa al nivel de $\mathrm{p}<0,05$ en la creencia irracional: evitación de problemas, donde el grupo de edades de 20 a 29 presenta el mayor rango promedio en comparación con los otros grupos etarios. Esto puede explicarse debido a las diversas responsabilidades que asume este grupo etario, que, como afirman Papalia et al. (2009), para este grupo etario mantener una adecuada higiene mental suele tener prioridad.

Estos son los resultados registrados en el estudio, que no son definitivos, ya que existen ciertas limitaciones al querer utilizarlos como marco referencial a determinadas explicaciones; son, en todo caso, aproximaciones relacionadas con las tendencias evidenciadas en cada una de las diez creencias aquí consideradas en estudiantes de un CEBA en el contexto educativo peruano.

\section{REFERENCIAS}

Carbonero, M. Á., Martín, L. J. y Feijó, M. (2010). Las creencias irracionales en relación con ciertas conductas de consumo en adolescentes. European Journal of Education and Psychology, 3(2), 287-298. http://www.redalyc.org/articulo. oa? id=129315468011

Cartagena,N.V.(2011). Errores típicos de pensamiento en adolescentes de 11 a 16 años de edad con alteraciones del autoestima que acuden al Instituto Médico Tierra Nueva en el periodo 20102011 [Informe Final de Trabajo de Titulación, Universidad Central del Ecuador]. http://www. dspace.uce.edu.ec/bitstream/25000/1406/1/TUCE-0007-31.pdf
Caso, Y. P. (2018). Creencias irracionales y conducta agresiva según género en estudiantes de Instituciones Educativas Públicas en San Juan de Lurigancho. Lima, 2018 [Tesis de licenciatura, Universidad César Vallejo] https://repositorio.ucv. edu.pe/bitstream/handle/20.500.12692/30220/ Caso_SY.pdf?seque nce $=1 \&$ isAllowed $=\mathrm{y}$

Castillo, I. E. (2017). Agresividad y creencias irracionales en los alumnos de $4^{\circ}$ y $5^{\circ}$ de secundaria en Instituciones Educativas Públicas del distrito de Puente Piedra, 2017 [Tesis de licenciatura, Universidad César Vallejo]. https://repositorio. ucv.edu.pe/bitstream/handle/20.500.12692/3206/ Castillo_AIE.pdf?seq uence $=1 \&$ isAllowed $=\mathrm{y}$

Ellis, A. (1998). Teoría y práctica de la terapia racional emotivo-conductual. Siglo Veintiuno.

Ellis, A. y Grieger, R. (1981). Manual de terapia racional-emotiva. Desclée de Brouwer.

Kuba, C. M. (2017). Relación entre creencias irracionales y ansiedad social en estudiantes de la facultad de psicología de una universidad privada de Lima Metropolitana [Tesis de licenciatura, Universidad Peruana Cayetano Heredia]. http:// repositorio.upch.edu.pe/handle/upch/659

López, E. I. (2015). Ideas irracionales y su influencia en las conductas agresivas de los estudiantes de los primeros cursos de bachillerato en los colegios fiscales urbanos del cantón Pelileo de la provincia de Tungurahua [Tesis de licenciatura, Universidad Técnica de Ambato]. http://repositorio.uta.edu.ec/ bitstream/123456789/9319/1/L\%C3\%B3pez\%20 Manjarr\%C 3\%A9s\%2C\%20Evelin\%20Isabel. pdf

McKay, E. y Navas, R. (2008). Inventario de creencias irracionales. Psichomethric.

Olaya,T.M.,Ruíz,L.M.y Sosa,S.(2014).Relaciónentre ideas irracionales, agresividad e ira en estudiantes de bachillerato de la Institución Educativa de Formación para la vida CEVIDA del municipio de Bello [Tesis de licenciatura, Corporación Universitaria Minuto de Dios Seccional Bello]. https://repository.uniminuto.edu/bitstream/ handle/10656/4175/TP_OlayaTatiana_2014.pd $\mathrm{f}$ ?sequence $=1$ \&isAllowed $=\mathrm{y}$ 
Otero, V. (2001). Convivencia escolar problemas y soluciones. Complutense de Educación, 12(1), 295-318. http://revistas. ucm.es/index.php/ RCED/article/view/RCED0101120295A/16905

Papalia, D., Wendkos, S. y Feldman, R. (2009). Desarrollo humano. McGraw-Hill.

Pervin, L.A. (1996). The science of personality. Wiley.

Ramírez, T. (1997). Cómo hacer un proyecto de investigación. Panapo.

Rivas, M. S. (2016). Relación entre clima social familiar y creencias irracionales en estudiantes del 5to grado de nivel secundario de la I.E. Enrique López Albújar. Piura 2015 [Tesis de licenciatura, Universidad Católica Los Ángeles de Chimbote]. http://repositorio.uladech.edu.
pe/bitstream/handle/123456789/131/RIVAS NIZAMA_M AHLI_SARAI_CLIMA_SOCIAL_ FAMILIAR_CREENCIAS_IRRACIONALES. pdf?sequence $=1 \&$ is Allowed $=y$

Sánchez, H. y Reyes, C. (2015). Metodología y diseños en la investigación científica. Business Support Aneth.

Tang, J. C. (2011). Creencias irracionales en adolescentes infractores privados de libertad por delito sexual de un centro juvenil de varones de Lima [Tesis de licenciatura, Universidad CésarVallejo]. https://es.scribd.com/ doc/136587238/tesis-creencias-irracionales-2011

Fecha de recepción: 26 de abril 2021

Fecha de aceptación: 12 de mayo 2021 